\title{
REVISTA DE HUMANIDADES Y CIENCIAS SOCIALES
}

número 22 | julio-diciembre 2018 online ISSN 0719-3696 / ISSN 0718-655x

Alejandra Castillo

Sayak Valencia

valeria flores

María Belén Rosales

Marina Alvarado

Natalia Fischetti

Panchiba F. Barrientos

Márgara Millán

Verónica Schild

Luna Follegati Montenegro

Valentina Stutzin

Lieta Vivaldi

Nicolás Ried

\section{INTRODUCCIÓN}

Feminismos en América Latina. Introducción

Feminisms in Latin America. Introduction

\section{Artículos}

El Transfeminismo no es un Generismo

Transfeminism Is not a Genderism

Febriles alquimias del cuerpo. Una poética excrementicia

Febrile Alchemy of the Body. An Excremental Poetics

Ciberactivismo: praxis feminista y visibilidad política en \#NiUnaMenos Cyber-Activism: Feminist Praxis and Political Visibility in \#NiUnaMenos

Feminismos del Sur. Alusiones / Elusiones / Ilusiones

Southern Feminisms. Allusions / Elusions / Illusions

Sexo, género y mujeres: tensiones y quiebres desde la filosofía feminista Sex, Gender, and Women: Tensions and Disruptions from the Feminist Philosophy

La eclosión del sujeto del feminismo y la crítica de la modernidad capitalista The Eclosion of Feminism's Subject and the Critique of Capitalist Modernity

\section{ENTREVISTA}

Contingencia, democracia y neoliberalismo: reflexiones y tensiones a partir del movimiento feminista en la actualidad

Contingency, Democracy, and Neoliberalism: Reflections and Tensions from the Feminist Movement Today

\section{RESEÑAS}

Judith Butler, Zeynep Gambetti y Leticia Sabsay, eds. Vulnerability in Resistance. Durham NC: Duke University Press, 2016. 352 pp. ISBN 9780822362906

Judith Butler. Notes Toward a Performative Theory of Assembly.

Cambridge MA: Harvard University Press, 2015. 248 pp. ISBN 9780674967755 


\title{
Contingencia, democracia y neoliberalismo: reflexiones y tensiones a partir del movimiento feminista en la actualidad.
}

\author{
Verónica Schild \\ UNIVERSIDAD DE WESTERN \\ Luna Follegati Montenegro ${ }^{2}$ \\ Universidad de Chile
}

Recibido: 13 de junio de 2018

Aceptado: 27 de junio de 2018

\section{Resumen}

El análisis del movimiento feminista en los últimos treinta años se ha vuelto ineludible en el contexto latinoamericano actual, plagado de escenas feministas disruptivas en relación al relato oficial del movimiento. Con esto surge la necesidad de comprender críticamente su pasado, los procesos de institucionalización y la agenda de género implementadas en los 90', como también aprehender nuevas herramientas y cruces teóricos que den cuenta de las necesidades actuales del movimiento. Verónica Schild, académica e investigadora feminista, entrega una lectura punzante en relación a tres ejes que parecen inevitables en el desarrollo de un pensamiento feminista actual: comprender su densidad y diversidad en contraposición a una lectura única u homogénea; tensionar el cruce particular entre neoliberalismo y transición democrática desde una analítica feminista; y, por último, comprender los efectos actuales de las políticas estatales en relación a la mujer, familia y cuidado. Schild ofrece una lectura que increpa -desde el feminismo- al modelo capitalista de despojo, planteando su límite social y ecológico en contextos donde comunidades enteras son afectadas, acentuando su violencia en espacios rurales y comunidades indígenas y particularmente en las mujeres y sus cuerpos. Como respuesta, Schild se pregunta por las posibilidades actuales de repensar las claves

1 Profesora emérita de la Universidad de Western (Ontario, Canadá). Correo electrónico: vschild@uwo.ca.

2 Candidata a doctora en Filosofía Política por la Universidad de Chile (Santiago, Chile). Correo electrónico: 1follegati@gmail.com. 
Contingencia, democracia y neoliberalismo: reflexiones y tensiones a partir del movimiento feminista en la actualidad.

teóricas del feminismo, visitando para ello los recursos de la tradición feminista del marxismo y socialismo.

\section{Palabras clave}

Feminismo, Neoliberalismo, Transición a la democracia, Género, Izquierda. 


\title{
Contingency, Democracy, and Neoliberalism: Reflections and Tensions from the Feminist Movement Today.
}

\begin{abstract}
The history and analysis of the feminist movement in the last 30 years has become unavoidable in the current Latin American context, fraught with feminist scenes that are disruptive in relation to the official narrative of the movement. With this, the need arises to critically understand the past of the movement, the processes of institutionalization, and the gender agenda implemented in the 90', as well as to develop new tools and theoretical analyses that account for the current needs of the movement. In the wake of these phenomena, Veronica Schild, a feminist academic and researcher, offers a critical reading related to three axes inevitable for the development of a current feminist thought: to understand the density and diversity of feminism as opposed to a single, homogeneous reading; the particular cross between neoliberalism and transition to democracy from feminist analyses; and finally, to understand the current effects of state policies related to women, family, and care. Schild's perspective transversally establishes a reading that remarks -from feminism- the capitalist model of dispossession, raising its social and ecological limits in contexts in which entire communities are affected, accentuating its violence in rural spaces and indigenous communities, particularly for women and women's bodies. As a response, Schild asks about the current possibilities of rethinking the theoretical keys of feminism, discussing the resources of the feminist tradition of Marxism and socialism.
\end{abstract}

\section{Keywords}

Feminism, Neoliberalism, Transition to democracy, Gender, Left. 


\section{Introducción}

Revisitar actualmente la crítica e investigación a Verónica Schild es un gesto importante en dos sentidos. En primer lugar, la analítica de Schild ha tenido una singular relevancia en relación a los procesos de institucionalización del feminismo en Chile, dando paso a una lectura crítica en relación al devenir de los estudios de género en nuestro país. En segundo lugar, la posibilidad de mantener un vínculo sustantivo con la experiencia chilena - pero desde una voz planteada fuera del país- la posiciona desde una crítica aguda a la forma en que el neoliberalismo ha permeado los distintos espacios, subjetividades y escenarios chilenos.

De formación inicial en filosofía, y luego doctora en Ciencia Política, Verónica Schild ha mantenido un constante contacto e investigación en relación al feminismo en Chile. Su llegada a este país en 1986 -luego de una estancia en Estados Unidos y Canadá, donde finalmente se radicó-la llevan a emprender su investigación doctoral en los sectores populares de Santiago, énfasis inicial que sin embargo mantuvo insistentemente durante las dos décadas posteriores. Mediante estadías prolongadas en La Pintana, comenzó un proceso investigativo sobre las organizaciones de mujeres populares y su relación con el movimiento de mujeres y, sobre todo, con el feminismo de la década de los ochenta que emergía en el contexto de la dictadura. A partir de esto, genera una sustantiva producción sobre movimientos sociales ${ }^{3}$, neoliberalización de estado y nuevas ciudadanías ${ }^{4}$, manteniendo una elaboración intelectual que se erigió al margen de las lecturas e investigaciones de la academia chilena vinculada a los estudios de género, ciertamente institucionalizados.

Hoy, con un movimiento feminista estudiantil a cuestas, la necesaria lectura de su obra se vuelve fundamental para recomponer un pensamiento feminista crítico que pueda destrabar los entuertos de la década de los noventa, poniendo atención en las implicancias de la dimensión de género para la construcción de un estado social neoliberal ${ }^{5}$. Particularmente, resalta la relación que establece entre un feminismo institucionalizado que potenció programas de superación de la pobreza con enfoque de género, la "feminización del Estado". Su visión, de carácter estructural y situada, nos entrega elementos fundamentales para hacer frente a la neoliberalización de la agenda de género nacional, como también para acentuar una

3 Véase Verónica Schild, "Recasting 'Popular' Movements: Gender and Political Learning in Neighbourhood Organizations in Chile", Latin American Perspectives 21 (1994): 59-80.

4 Verónica Schild, “¿Nuevos sujetos de derechos? Movimientos de mujeres y la construcción de ciudadanía en las 'nuevas democracias'", en Política cultural \& cultura política: una nueva mirada sobre los movimientos sociales latinoamericanos, eds. Arturo Escobar, Sonia Álvarez y Evelina Dagnino (Bogotá: Taurus-ICANH, 2001), 119-146.

5 Véase Verónica Schild, "Los feminismos y la construcción del estado social neoliberal en América Latina", en Desigualdades en un Mundo Globalizado, ed. Narda Henriquez, Gerardo Damonte, Marianne Braig y Barbara Göbel (Lima: CISEPA, Pontificia Universidad Católica del Perú, 2015), 75-100. 
lectura feminista que retoma elementos críticos provenientes del marxismo. Por más de 30 años, las investigaciones de Schild han compartido ese cruce ineludible para un pensamiento y acción feminista: cómo hacer frente desde un "feminismo crítico" a las convergencias entre el proyecto feminista y el neoliberal, y sus implicancias ${ }^{6}$. La entrevista comprende estas intersecciones, desde la contingencia del movimiento feminista estudiantil hacia un énfasis más transversal que cuestiona la relación entre neoliberalismo y democracia, y con ello, el rol de la izquierda y los partidos en la configuración del movimiento feminista. Hoy en día, leer sus textos se vuelve un ineludible si deseamos destrabar las claves analíticas con que se ha mirado y comprendido el género, el feminismo y su movimiento.

\section{La contingencia del movimiento feminista en la actualidad}

Luna Follegati (L): Actualmente nos encontramos en un escenario en que el movimiento feminista - a lo menos en América Latina - se ha vuelto a posicionar como una actora importante, luego del impulso de la década de los ochenta. En este sentido, se vuelve inevitable comenzar el recorrido consultándote sobre tu visión respecto de las últimas movilizaciones que se han generado en el Cono Sur. Por ejemplo, Ni Una Menos, las marchas por el aborto libre y el movimiento feminista estudiantil en Chile, han generado un importante impacto tanto en la agenda mediática como en la sociedad en general. A partir de lo anterior, ¿Qué elementos o ejes vez como continuidades y discontinuidades entre ambos procesos?

Verónica Schild (V): Más allá del mensaje obvio de esta nueva irrupción feminista, me parece que lo que su rabia colectiva - casi de un modo visceral- nos ayuda a entender, es que hay una brecha tremenda entre, por un lado, un discurso de derechos de la mujer, de la equidad de género, del derecho a vivir sin violencia, de un sin fin de temas que han sido instaladas en las últimas décadas por los medios y los pronunciamientos de distintos gobiernos en el lenguaje de programas sociales, el de las ofertas comerciales y bancarias, etcétera, y por el otro la institucionalización real y por ende la perpetuación en el tiempo de estos derechos. Con esto quiero decir, que hay mucho discurso de empoderamiento e igualdad, algunos logros importantes que hay que reconocer, y hasta una sensibilidad en el lenguaje público que hace resaltar - y también rechazar- las groserías que antes se toleraban como normales. Pero de ahí a decir que, como intento de transformar el contexto en que la sociedad chilena funciona y llegar a ser parte de un sentido común institucional,

6 Verónica Schild, "Care and Punishment in Latin America: The Gendered Neoliberalization of the Chilean State", in Neoliberalism Interrupted: Social Change and Contested Governance in Contemporary Latin America, eds. Mark Goodale and Nancy Postero (Stanford: Stanford University Press, 2013), 195-224. 
esto no sucede ni en este país, ni en la región en general. Las políticas en beneficio de las mujeres en Chile malamente se han institucionalizado.

Por supuesto que ha habido logros, pero los logros siempre generan expectativas y yo diría que lo que estamos presenciando hoy día es precisamente promesas de igualdad de derechos que no han llegado a instalarse. Ahora, no estoy para nada de acuerdo con aquellos en la política que ven a Chile en buen camino hacia la modernidad, y que imploran paciencia. En boca de los sectores de la centroizquierda que gobernó este país por décadas, eso me parece de una profunda falta de honestidad intelectual -por decir lo menos-, pero también política. Aquí incluyo también a sectores de un feminismo institucionalizado que defiende esos logros como primeros pasos. Yo diría que habría que empezar por aceptar que vamos por un rumbo que comparte tendencias pero que tiene sus propios bemoles, y aquellos que hemos estudiado el tema de la democracia en América Latina desde la época de las dictaduras "modernas" sabemos que el problema de la inhabilidad política de institucionalizar cambios, y por ende la debilidad institucional de estos, ¡es permanente! Seguir insistiendo en una historia de origen que no fue tal, porque los sistemas democráticos se instalaron en contextos poscoloniales que perpetuaron jerarquías sociales brutales, y que se siguen reproduciendo - caso específico es la

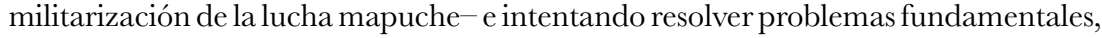
como la relación con los pueblos originarios, por medio del uso de la violencia, ja pesar de tratados y acuerdos internacionales! Entonces, el proyecto compartido de la política impone una nueva visión exitista, que es además clasista y racista, de la casi llegada a la tan codiciada "modernidad". A mi modo de ver, esto es perpetuar en el tiempo la imposibilidad de institucionalizar cambios reales y buscar otro modo de convivencia.Quisiera añadir que lo que me preocupa de la construcción mediática y política de las movilizaciones feministas actuales es el peligro de construir nuevamente al feminismo como actor único, e invisibilizar las voces que anclan sus demandas en los contextos brutales de la vida cotidiana de la mayoría. Qué quiero decir con esto: el caracterizar la irrupción feminista como "actor importante" invisibiliza su diversidad y complejidad e instala una visión homogénea que, en el fondo, se basa en la experiencia de jóvenes urbanas y de clase media como "el" referente político con quien dialogar. Me parece que debemos ser cuidadosas, sobre todo como feministas críticas, ya que revisando las declaraciones y conociendo la diversidad de organizaciones y luchas que han surgido en este país y en el resto de América Latina por los últimos 30 años, queda muy claro que hoy como nunca el actor feminista es múltiple. Pensando en el caso de Chile, por ejemplo, las recientes marchas han unido en la calle a estos feminismos bajo el interés común de decir basta de violencia, maltratos, y control de nuestra autonomía sexual y reproductiva, y de exigir que de una vez por todas asumamos la responsabilidad social, política e institucional del derecho de las mujeres a vivir una vida libre de violencias. Pero por 
debajo de estas convergencias y alianzas políticas hay realidades muy distintas que proponen demandas, proyectos e intervenciones, en nombre de feminismos propios. Si durante los ochenta, cuando me tocó participar en iniciativas y discusiones sobre feminismo popular (urbano), estas eran descartadas por una mirada feminista única instalada en centros de investigación y de activismo de clase media, caer en esto hoy día sería un grave error. En los ochenta, los talleres, fondos, y vínculos con las ONGs facilitaron los recursos para pensar en otros feminismos.

$\boldsymbol{L}$ : Con respecto a esto último, ¿Consideras que existen feminismos diversos, proyectos que divergen entre sí? ¿cuáles serían?

V: Hoy día, una nueva generación se nutre de herramientas de análisis y conceptos que circulan por redes transnacionales propias. Podría nombrar algunas experiencias, como la organización Asamblea de Mujeres de la Bandera que se apoya en el concepto de territorio para desarrollar "un feminismo diferente" con solido arraigo social, así como las activistas del Movimiento de Pobladoras y Pobladores por la Dignidad (MPD) que vinculan la lucha por la vivienda digna con el feminismo, y se apoyan en elementos del feminismo comunitario de Bolivia. Pienso también en la Asociación Nacional de Mujeres Indígenas y Rurales (ANAMURI), una organización con reconocimiento internacional que este año celebra 20 años de existencia y que reúne a mujeres campesinas y de pueblos originarios de todo el país en la defensa de territorios y sus derechos "como mujeres campesinas y pueblos originarios". La Asociación Nacional de Mujeres Rurales e Indígenas ANAMURI pertenece a una red mundial que se plantea "en lucha en contra del capitalismo y el patriarcado y por la defensa de la producción campesina que nos permita avanzar hacia una reforma agraria integral con soberanía alimentaria y popular"". Hoy como nunca, entonces, necesitamos pensar en las alianzas feministas y un paso importante es luchar contra la discriminación que existe en el propio movimiento. Esto es algo que activistas mapuche reclaman con mucha fuerza, como bien aclaran Millaray Painemal e Isabel Canet ${ }^{8}$.

L: Uno de los aspectos que se han relevado en los movimientos recientes dice relación con la necesidad de repensar los marcos teóricos y políticos desde los cuales se ha teorizado el feminismo. El movimiento feminista estudiantil de Chile ha ensayado distintas corrientes de feminismo - desde el separatismo al feminismo socialista-, mostrando una pluralidad de visiones en disputa. En este

7 ANAMURI, "Anamuri: Celebrando 20 años de unidad y lucha de las mujeres del campo", Medio a Medio, 13 de junio de 2018. Consultado en agosto de 2018, disponible en_http://www. agenciadenoticias.org/anamuri-celebrando-20-anos-de-unidad-y-lucha-de-las-mujeres-delcampo/.

$8 \quad$ Millaray Painemal e Isabel Cañet, “¿Es que acaso debemos ser todas feministas? Reflexiones de mujeres mapuche para un debate", El Desconcierto, 04 de marzo de 2018. Consultado el 13 de agosto de 2018. Disponible en http://www.eldesconcierto.cl/2018/03/04/es-que-acasodebemos-ser-todas-feministas-reflexiones-de-mujeres-mapuche-para-un-debate/. 
sentido, y atendiendo a la experiencia de los noventa, ¿qué aspectos consideras pertinentes rescatar de la trayectoria histórica de la teoría y movimiento feminista del siglo XX?

$\boldsymbol{V}$ : Esta pregunta es muy interesante. Rescatar trayectorias significa inevitablemente posicionarse políticamente y también concretamente en el momento histórico en que las luchas feministas actuales están insertas. A mi modo de ver, este es un momento crítico en nuestras sociedades, que nos obliga a considerar el contexto en que se insertan las movilizaciones. Lo más urgente, a mi juicio, es reconocer que más allá del entorno urbano de los debates y muchas de las iniciativas, está la realidad de los límites sociales y ecológicos de un modelo capitalista de despojo que tiene impactos brutales para las comunidades afectadas, muchas de ellas rurales e indígenas, y que recaen sobre todo en las mujeres. En Chile, por mencionar dos ejemplos dramáticos, con un sistema de privatización del agua único en el mundo, donde su acceso no está garantizado como derecho humano básico, comunidades enteras están quedándose sin agua. A esto se le suman comunidades urbanas y rurales que concentran niveles de contaminación química que violan los estándares internacionales, con impactos serios para la salud de comunidades enteras, pero que se consideran inevitables "zonas de sacrificio" por el bien nacional. Estas son realidades que se multiplican a lo largo del país. No es coincidencia, entonces, que las mujeres estén a la vanguardia de estas importantes luchas. A mi parecer, estas luchas son un eje central de la lucha contra un capitalismo neoliberal y patriarcal, y por un mundo distinto, pero más allá de la presencia de las organizaciones en las marchas del 8 de marzo, esta realidad pareciera no existir para un feminismo de orientación urbana. ¿Cómo incorporar en nuestras reflexiones y prácticas colectivas una reflexión sobre la magnitud de los cambios en curso y convencernos como feministas que los problemas ecológicos y del medio ambiente no son externos al feminismo? Esa es mi gran preocupación actual.

Volviendo a la pregunta, un punto de partida necesario está en rescatar, valorar y renovar recursos de una tradición feminista marxista/socialista, porque nos ayudan a entender la relación estructural entre el capitalismo y las opresiones específicas de las mujeres, y así, anclar nuestro proyecto en un análisis más amplio. Curiosamente, este rescate de una tradición crítica es algo que ya está sucediendo mundialmente. Mi punto de referencia para pensar en esto son las movilizaciones del 8 de marzo en toda la región, que se proponen cada vez con más claridad el fuerte deseo de sumar fuerzas muy diversas y formar alianzas, feministas y con otros movimientos sociales, para protestar contra las múltiples opresiones de un capitalismo neoliberal en crisis. Me enfoco más bien en ello, que me parece más representativo del conjunto de demandas feministas en Chile que el movimiento feminista estudiantil, una bocanada de aire fresco con demandas importantes y que me llama mucho la atención, pero que veo como algo netamente urbano. Indudablemente que este mayo chileno marca un hito importante, pero es también más acotado en su 
impacto. Me parece, entonces, que este es un momento propicio para reflexionar sobre los elementos necesarios para entender los contextos más amplios en que están insertas estas nuevas organizaciones y luchas feministas, y también para volver a la tarea pendiente que es evitar caer en conceptos reduccionistas del sujeto político feminista que minimicen luchas que hoy se han vuelto críticas. Entonces, como sugerí anteriormente, rescato de la experiencia de los 80 y 90 la tarea pendiente de pensar en política feminista más allá de las discriminaciones y silenciamientos que llevaron a conformar un pensamiento único. Rescatar trayectorias significa posicionarse políticamente y a mi modo de ver, este es un momento crítico en nuestras sociedades y ha llegado la hora de recuperar recursos de una tradición feminista marxista/socialista. ¿Qué sucede en los contextos donde están insertas las mujeres organizadas que las impulsan a salir a la calle y proponerse la necesidad de formar alianzas? Necesitamos elementos que nos ayuden a posicionar luchas por la autonomía reproductiva y sexual en el contexto más amplio del modelo de desarrollo imperante.

L: Históricamente, una de las tensiones que me parece interesante revisitar es la relación entre feminismo y partidos políticos. Atendiendo a la actualidad de un movimiento que se vincula críticamente con las formas tradicionales de la política, pero a la vez muy presente en espacios politicos emergentes, particularmente en el caso de Chile al interior de los partidos que conforman el Frente Amplio, por ejemplo, ¿cómo vez esta relación en la actualidad? ¿Qué aspectos se deben rescatar de la relación entre feminismo e izquierda, y más particularmente, entre feminismo y partidos políticos?

V: Para empezar, diría que me complica entender la relación entre "feminismo" e izquierda, precisamente por lo que mencioné anteriormente. Son varios los feminismos y el tema para mí sería cuál es la posición de un feminismo de izquierda dentro de esta izquierda emergente. El hecho de que mujeres de partidos de derecha se identifiquen como feministas - icon todas las contradicciones que eso conlleva!me parece que hace necesario especificar de qué feminismos estamos hablando. Ahora bien, a un nivel muy global, desde una perspectiva amplia de género, queda muy claro que todos los espacios institucionales, desde la universidad hasta las instancias de gobierno, el sistema judicial, y para que decir los espacios políticos como partidos, parlamentos, etcétera, siguen siendo espacios masculinizados. Y esto es algo mundial que obliga a las mujeres que se instalan en ellos a una lucha permanente. Las expresiones de apoyo de mujeres de partidos de derecha responden más bien a esa lucha, y dan cuenta también del grado en que se ha instalado una nueva sensibilidad a nivel social que permite ponerle nombre a este malestar que nos toca vivir a todas en los distintos ámbitos del espacio público. 


\section{Democracia y feminismo}

$\boldsymbol{L}$ : Los años noventa fueron claves en el fortalecimiento del neoliberalismo en la región, cuestión que también tensionó al feminismo y sus estrategias de acción. En este sentido, cabe preguntarse frente a los desafios del movimiento en el presente, ¿cuáles serían aquellos enclaves o nudos en que el neoliberalismo establece una relación con el feminismo?

V: Para empezar, quisiera aclarar que después de varias décadas de reestructuración del modelo de acumulación capitalista en la región, y de las profundas transformaciones sociales y ecológicas que ha producido, es evidente que la política neoliberal contempla mucho más que las así llamadas modernizaciones económicas e institucionales implementadas con apoyo de agencias como el Banco Mundial, el BID y el Fondo Monetario Internacional. El neoliberalismo es un proyecto de dominación política cuyo fin es lograr la adaptación de nuestras sociedades a una economía liberada de responsabilidades sociales. Como bien dice Wendy Brown, apoyándose en Foucault, "el neoliberalismo es un modo distintivo de la razón, de la producción de sujetos, una "conducta de la conducta", y un esquema de valorización ${ }^{9}$. Para concretar esta noción cultural y política del neoliberalismo, sugiero pensar la racionalidad neoliberal como una gramática que nos ofrece un lenguaje específico, y que por definición también delimita los parámetros de lo posible. Esta perspectiva es clave para entender los "nudos" en que el neoliberalismo establece una relación con el feminismo, entendido en su materialidad, como procesos encarnados. Quisiera destacar que al referirnos a los "nudos", lo que se trata - a partir de lo que he venido investigando sobre el caso chileno por casi tres décadas - es estudiar la convergencia entre proyectos neoliberales y ciertas prácticas y discursos feministas. Abordar este tema significa reconocerlos como procesos situados: a partir de relaciones que se tejen entre feministas, sus agendas y sus opciones políticas, con las instituciones y agencias del estado, y con ese universo de mujeres de las cuales se tornan en voceras autodesignadas. Hacer política feminista en la medida de lo posible, a partir de 1990, incidir pragmáticamente sobre todo en el área de la política social y de una agenda de derechos delimitada, fue la opción de un sector muy visible del feminismo, pero no único. Hay que reconocer de que esta política pragmática estuvo enmarcada -desde un inicio- en una supuesta transición a la democracia que tuvo como su condición la de ser continuidad de un modelo de acumulación capitalista tremendamente excluyente, impuesto durante la dictadura cívico-militar. Cabe recordar que generalizamos al hablar de "el" feminismo chileno, y nos olvidamos de aquellas voces que se quedaron fuera, pues no todos los feminismos chilenos optaron por institucionalizar sus agendas. Habría que preguntarse el cómo y el porqué de este olvido, y es algo que me planteé en

9 Wendy Brown, Undoing the Demos. Neoliberal's Stealth Revolution (Nueva York: Zone Books, 2015), 21. 
investigaciones en los noventa, que muestran que esta institucionalización de cierto feminismo resultó no solo en la automarginación de aquellas que se definieron como autónomas, sino que también en el silenciamiento e invisibilización de un incipiente feminismo popular ${ }^{10}$. La así llamada institucionalización del feminismo en los noventa, con sus consiguientes exclusiones, es por lo demás un fenómeno que se repite en toda la región.

L: Un punto interesante, y relevante de destacar para el caso de la región, es el cruce entre neoliberalismo y procesos de transición a la democracia. Efectivamente la historia política e institucional de la región da cuenta de eso; sin embargo, también es usual que dicha lectura omita las agendas de género. Me parece interesante atender a cómo las políticas de la transición reconocen dos ejes: por una parte, una acción efectiva en relación a los movimientos sociales, y por otra, una política bajo la racionalidad neoliberal, como señalabas anteriormente. En este sentido, surgen algunas preguntas: ¿Cuáles serían las deudas de la democracia - en el contexto de la transición en Chile- en relación al movimiento feminista? ¿Cómo ves esa relación entre democracia, transición y feminismo?

V: Para comenzar, habría que preguntarse qué entendemos por "democracia". Desde un punto de vista de la teoría, la democracia es un concepto ambiguo, como nos recuerda la rica literatura reciente, por ejemplo, los trabajos de Giorgio Agamben, Jacques Ranciereo Etienne Balibar. Más allá de las figuras normativas, sus promesas y sus limitaciones, lo que me interesa es plantear esta pregunta desde la experiencia histórica y estructural de nuestra región. Como sostuve en algún momento, al celebrar las posibilidades para la acción feminista de una "vuelta" a la democracia, muchas, pero no todas, olvidaron su carácter de democracia capitalista. Hoy asistimos a una crítica abierta de nuevas generaciones a eso que en su momento la feminista peruana Maruja Barrig llamo los "malestares del feminismo"11 y a una urgente búsqueda de alternativas a las sociedades excluyentes que se han consolidado en toda la región. Las varias décadas de un modelo capitalista arrasador han moldeado los contornos de sociedades que, como bien lo plantea Maristella Svampa, están estructuradas sobre "la base de la cristalización de las desigualdades tanto económicas como sociales y culturales" "12. También urge reconocer que, a diferencia de otras épocas, este modelo destructivo ha resultado de una acumulación de daños ecológicos de impacto profundo y desigual. Nuestra reflexión sobre las deudas de la transición y la democracia con las demandas feministas debe situarse en ese contexto más amplio. Deberíamos partir por reconocer que ya estamos resituadas en una perspectiva de derechos que es el fruto de los cambios institucionales y políticos de las últimas décadas, y que en el caso chileno está garantizada por la Constitución (neoliberal)

10 Schild, “Nuevos sujetos de Derechos?”

11 Maruja Barrig, "Los malestares del feminismo latinoamericano: una nueva lectura”, ponencia presentada en la conferencia LASA, 1998. Manuscrito.

12 Maristella Svampa, La sociedad excluyente: La Argentina bajo el signo del neoliberalismo (Buenos Aires: Editorial Taurus, 2005), 12. 
de 1980. En este contexto, los nuevos espacios de derecho creados por los distintos Planes de Equidad de Género y los compromisos internacionales firmados por los gobiernos de nuestra región desde la reunión en Beijing de 1995 se conforman a través de un discurso liberal y limitado de derechos, que no permite establecer un sistema de derechos universales para todas (y todos). De hecho, las luchas feministas de las últimas décadas han sido por la conquista de una serie de derechos que las haga más iguales como ciudadanas, sin cuestionar o desafiar el modelo dominante de ciudadanía y política. Ahora bien, reconocer esto nos permite por ejemplo comprender por qué avances en ciertos derechos de las mujeres (y de las así llamadas disidencias sexuales) no van acompañados de logros sociales y económicos básicos que permitan la vida digna a la mayoría, y se dan en contextos donde persiste una profunda desigualdad y precarización de la vida tanto laboral como social, a lo que se le suma, como nunca antes, el impacto del daño ecológico que ya mencioné.

Yo creo que la democracia actual, vinculada a la defensa del libre mercado y a un discurso limitado y mercantilizado de derechos individuales, establece los parámetros de lo "decible", y claramente permite ciertas reivindicaciones, pero excluye otras. Es decir, una serie de demandas por derechos socioeconómicos y ecológicos que tienen que ver con la justicia social y económica, incluyendo la dimensión ambiental y justicia ecológica que se torna cada vez más urgente, no tienen cabida. La deuda tiene que ver con esto, con una democracia que, como tan bien nos ilustra el caso chileno, permite la vulneración de las condiciones de vida de comunidades enteras y afecta dramáticamente la calidad de vida de la mayoría de los ciudadanos y ciudadanas, con efectos especialmente perniciosos para las mujeres.

L: Uno de los aspectos claves de los procesos de transición a la democracia-particularmente en el caso de Chile - tuvo que ver con la segmentación del tejido social, particularmente a través de políticas que prefiguraron una recomposición de las formas sociales en el contexto de la resistencia al periodo de la dictadura. Las organizaciones, colectivos o espacios de organización feministas se vieron fuertemente afectados en los noventa. En este sentido, címo la democracia chilena -en el contexto de la transición - influiría en un proceso de segmentación o individuación de la demanda feminista?

$\boldsymbol{V}$ : Mis investigaciones sobre la construcción de nuevos ciudadanos bajo el signo neoliberal, insisten en considerarla como procesos encarnados y por lo tanto con una fuerte dimensión de género, y resaltan el énfasis que se le da a la dimensión de las responsabilidades sobre los derechos ciudadanos, apoyándose en un alcance al concepto de responsabilidad claramente diferenciado y basado en supuestos heteronormativos. Desde mi perspectiva, este proceso de "responsabilizacion" diferenciada, como elemento fundamental de una nueva ciudadanía, esta emblemáticamente ilustrado en la revitalización de la acción social del Estado a partir de fines de los años noventa. Como sugiero en mis estudios sobre el Estado 
social neoliberalizado en Chile, en su acción se reafirma y renueva la lógica fundamental de relaciones contractuales que ha imperado en el quehacer social del Estado de las últimas décadas, y se mantiene un concepto individualista limitado de los derechos, con insistencia en la responsabilidad ciudadana. En consecuencia, el supuesto vuelco a una nueva social democracia no fue tal. La característica central del quehacer de este Estado social neoliberalizado sigue siendo su orientación basada en la racionalidad del mercado, no de los derechos ciudadanos universales, lo que se traduce en una continuación de la focalización. Entonces, el universo de beneficiarios se amplía a más quintiles, por dar un ejemplo, y se mantiene el principio de corresponsabilidad, expresado en las transferencias condicionadas de la asistencia y en otros tipos de subsidios. Todo esto apunta a que la lógica de las libertades neoliberales se mantiene y refuerza hasta el presente. Lo que también he recalcado en mis estudios es que la experiencia concreta de estrategias de inclusión social a través del consumo y endeudamiento, y de la formación de sujetos ciudadanos de mercado, deviene después de varias décadas en una nueva ciudadanía ${ }^{13}$. Este es un cambio fundamental e histórico que logra enredar a vastos sectores de la sociedad en relaciones contractuales, a partir de la responsabilidad individual, y desplazar aquel concepto de solidaridad social que en otro momento orientó la demanda por derechos universales a los que al Estado le correspondía dar respuesta. Deleuze llamó a esta transformación el proceso de adaptación de las sociedades a un orden neoliberal a partir de la "responsabilizacion" de los individuos. Así, suponer que el Estado tiene obligaciones históricas en relación a una perspectiva universal de derechos ciudadanos me parece que es confundir una expectativa normativa con la construcción en el tiempo de una relación específica entre una cierta forma de Estado y un cierto tipo de sujeto político.

L: Pensando en la deuda en términos del traspaso entre las demandas feministas de finales de los ochenta y lo que finalmente se termina concretando como la "agenda feminista" en los noventa, que en el caso de Chile significó una restricción importante en relación a las condiciones de posibilidad que daba cuenta la política de la transición, ¿cuáles serían las particularidades de las democracias latinoamericanas que frenan -o tensionan a lo menos- los avances de las temáticas o reivindicaciones de género?

$\boldsymbol{V}$ : Como ya mencioné, para poder entender el rol de la democracia en este caso, debemos detenernos en las características específicas de nuestro contexto estructural y las articulaciones locales de proyectos neoliberales. En relación a este último punto, nuestros proyectos neoliberales se conjugan de recursos culturales particulares, un catolicismo muy conservador en casos como el chileno, y vertientes igualmente conservadoras de un cristianismo evangélico en otros puntos de la región, que

13 Véanse Verónica Schild “Empowering Consumer Citizens or Governing Poor Female Subjects. The Institutionalization of 'Self-Development' in the Chilean Social Policy Field", Journal of Consumer Culture 7, no. 2 (2007): 179-203; también John Clarke, Changing Welfare, Changing States (Londres: Sage Publications, 2004). 
prometen no sólo limitar los avances en relación a derechos sexuales y reproductivos, sino que dar marcha atrás. Vale la pena recordar que este conservadurismo religioso se articula con un proyecto de desarrollo excluyente y su legitimación a partir de un discurso de derechos que aboga por las libertades individuales mercantilizadas, y que defiende un concepto de sociedad basado en la responsabilidad individual. Curiosamente, de liberal, en el sentido clásico, poco tiene esta mutación política latinoamericana, ya que un individualismo inédito, que se defiende como parte de la nueva modernidad, convive con un conservadurismo reaccionario. Es en este peculiar caldo de cultivo que debemos dimensionar "la democracia", porque por un lado pareciera ser liberal, pero obviamente no lo es cuando se trata de realmente abarcar los derechos individuales plenos de las mujeres. Volviendo a tu pregunta, sobre lo que decías en tu texto - que la transición pacta a través de las demandas y los cuerpos de las mujeres ${ }^{14}$ - creo que eso está muy bien ilustrado en el caso de países centroamericanos como Nicaragua y más recientemente Costa Rica, donde hubo avances en derechos sexuales y reproductivos, y ahora hay fuertes retrocesos.

Chile es un caso interesante. Es curioso cómo, en el contexto de la nueva ley de aborto en tres causales, se presenta a Chile como un país "un poco monje", como la Irlanda del Cono Sur, país muy católico, donde supuestamente jamás ha existido la posibilidad de aborto legal. Mientras tanto, el debate político interno por parte de la derecha y de sectores católicos de centro intentó convencer al país de que este era un tema nuevo, instalado sin dudas por sectores ultras. Esto, en consecuencia de que el aborto inducido habría alcanzado tales niveles que lo convirtieron en un problema epidemiológico para el país desde los años treinta del siglo XX. Se calcula, por ejemplo, que hasta 1968 por lo menos un tercio de la población femenina practicaba el aborto inducido, con altos índices de mortalidad materna. Habría que preguntarse, en efecto, ¿por qué esta amnesia histórica respecto a un tema que preocupó a sectores importantes de la política, del mundo profesional y del feminismo por décadas? Vale recordar el compromiso del Movimiento Pro Emancipación de la Mujer Chilena, MEMCH, fundado en 1935, de luchar por la emancipación jurídica, social y biológica de las mujeres ${ }^{15}$. No olvidemos que entre los años 1930 y 1989, el Articulo 119 del Código Sanitario permitía el aborto para resguardar la vida, integridad y salud de la madre, y que esta excepción fue

14 Luna Follegati, "El feminismo se ha vuelto una necesidad: movimiento estudiantil y organización feminista (2000-2016)", en Juventud y espacio en las Américas. I Taller Casa Tomada, comps. Ana Niria Albo y Camila Valdés León (La Habana, Editorial Casa de las Américas: 2016).

15 Claudia Rojas, “Poder, mujeres y cambio en Chile (1964-1973): un capítulo de nuestra historia”, tesis para optar al grado de magíster en Historia, Universidad Autónoma Metropolitana, 1994. 
eliminada en 1989 por Ley 18,826 ${ }^{16}$. Volviendo a mi punto inicial, creo entonces que, al plantear el tema de la democracia y su relación con las demandas feministas, hay que detenerse también en un análisis del factor religioso reaccionario como un componente valórico fuerte de los neoliberalismos latinoamericanos.

\section{Políticas neoliberales bajo el contexto democrático}

L: Continuando con lo que señalabas anteriormente, me gustaría que desarrollaras un poco más cómo las lógicas de trabajo neoliberal también se reproducen desde una perspectiva de género propiciada por el Estado. ¿Cómo ves la cuestión de la subjetividad neoliberal desde una perspectiva feminista? ${ }^{2}$ Cómo se vincula el problema del trabajo en este punto?

$\boldsymbol{V}$ : Cuando T.H. Marshall, a quien suele leerse en el debate latinoamericano sobre ciudadanía, propone un modelo "moderno" que identifica tres dimensiones de la ciudadanía - cívica, política y social-, plantea que cada una es un logro específico en el tiempo. Lo rescatable no es el modelo en sí, que cae en presunciones universalistas y, desde una perspectiva feminista, reproduce supuestos "masculinistas" del trabajo remunerado y no remunerado. Recordemos que, en esta historia de avances de derechos, los derechos sociales conllevan una sola responsabilidad de parte de los ciudadanos, y es la obligatoriedad del empleo masculino. El trabajo no remunerado que garantiza las condiciones de reproducción de la vida y de la sociedad en el tiempo, y que Marshall reconoce que recae sobre las mujeres, no es considerado como cumplimiento de una obligación ciudadana. Así, el efecto de esta teoría moderna de la ciudadanía es posicionar a la mujer como sujeto ciudadano de segunda clase. Ahora bien, Marshall nos deja una lección que sí es rescatable pero que fácilmente olvidamos, y es su perspectiva histórica de la ciudadanía. Según ésta, las identidades políticas surgen en relación con formas de Estado específicas, y en contextos altamente marcados por el conflicto y por relaciones desiguales de poder ${ }^{17}$. La conclusión que se puede sacar de esto es que la obligación social histórica del Estado fue el logro de pugnas políticas en un momento del capitalismo y de configuraciones de poder y de un campo político muy específicos y, a su vez, que éste es precisamente el desafio político que enfrentan los feminismos y las izquierdas emergentes en el presente, pero en un contexto histórico radicalmente distinto. El modelo de acumulación capitalista actual en nuestra región se basa en la superexplotación del trabajo tanto remunerado como no remunerado, en un incremento constante de formas de subempleo y desempleo, y de la feminización

16 Véase Hernán Pozo, "Mujeres Latinoamericanas en cifras, avances de investigación”, en VI. Situación Jurídica de la Mujer. Documento de Trabajo. Serie Estudios Sociales 16 (Santiago de Chile: FLACSO, 1991), 31-32.

17 Verónica Schild, “Neo-Liberalism's New Market Citizens: The Civilizing Dimension of Social Programs in Chile", Citizenship Studies 4 (2000). 
masiva del empleo precario. A diferencia de épocas anteriores, se suma hoy en día la acumulación de daños ambientales producto de la sobreexplotación de la naturaleza y que amenaza la sustentabilidad económica y social del modelo mismo. La lucha entonces por la restitución de derechos universales necesariamente va a tener que insertarse en un proyecto político más amplio y basado en un nuevo paradigma de la relación sociedad-naturaleza.

L: En Chile se han implementado una serie de políticas públicas con un enfoque de "género", que busca resolver ciertas problemáticas relativas a las tareas de cuidado, o de acompañamiento de la crianza. Por ejemplo, el programa "Mamá trabaja tranquila", "Chile crece contigo", o algunos aspectos del programa "Puente". De una u otra manera, el Estado intenta solventar o tratar de aminorar esta condición de que la mujer se hace cargo de la familia, del cuidado de los hijos. Estos programas tienen esa contradicción de individualizar en las mujeres una responsabilidad en relación al cuidado, pero también, esta situación es una realidad en el sentido de que por lo general son jefas de hogar o madres solteras que viven en condiciones de hacinamiento, y por lo mismo se ven beneficiadas con el acceso gratuito a salas cunas o jardines infantiles. ¿Cómo ves esas políticas de género en un contexto de políticas focalizadas? ¿Cuándo efectivamente "solucionan" problemas concretos o contingentes?

$\boldsymbol{V}$ : La pregunta es interesante porque, al dimensionar una crítica feminista al neoliberalismo, es importante no perder de vista la realidad de la mayoría de las mujeres. Obviamente que, en el contexto de un modelo de acumulación basado en la precarización económica y social, la política focalizada efectivamente da respuestas - yo no diría que "soluciona", pero da respuestas- a problemas concretos. Sin embargo, lo fundamental es que esta es una respuesta que no altera para nada el principio mercantilizado de esta asistencia: el acceso gratuito a salas cunas, jardines infantiles, subsidios a la vivienda y otros programas asistenciales, están enmarcadaos en un concepto de eficiencia y racionalidad de mercado que nada tiene que ver con los derechos de las mujeres, como derechos de todas. Aún más, las modificaciones que hizo el gobierno de la Nueva Mayoría (2006-2010) no alteraron esta racionalidad de mercado, sino que sencillamente la utilizaron para ampliar la llegada de beneficios a más quintiles. En ningún momento se transformó esta racionalidad en otra basada en derechos universales. Resulta entonces que este intento por solventar los problemas de "cuidado" no afecta ni siquiera a aquellas que viven en situaciones precarias y de bajos ingresos, pero que no son ni tan precarias ni de tan bajos ingresos. Esta lógica asistencial de mercado ha tenido un impacto fuerte en la solidaridad, pues genera divisiones y sospechas del otro, pero sí ha facilitado la inclusión mayor de mujeres al trabajo remunerado en las condiciones de precariedad imperantes. Pensando comparadamente en alternativas al tema del cuidado infantil, por ejemplo, el intento de países como Suecia en su apogeo social demócrata que ya no existe, fue de desmercantilizar el cuidado de los niños, lo que significa hacer al Estado, y no al mercado o las mujeres, responsable de 
crear las condiciones de acceso universal a guarderías, a pre y posnatal para ambos padres, así como obligaciones laborales dirigidas a empleadores explícitamente para no castigar laboralmente a los nuevos padres. Obviamente en las últimas décadas hemos asistido a cambios estructurales y a recomposiciones sociales y políticas, tanto locales como globales, que hacen impensable un cambio de esta índole.

L: Desde tus investigaciones ¿qué es lo hay detrás de las políticas de focalización, desde una perspectiva feminista?

$\boldsymbol{V}$ : Complementando lo anterior, las políticas de focalización asumen que el gasto social efectivo se dirige a las personas más necesitadas, bajo la lógica de la eficiencia de mercado. Pero esta lógica de mercado contradice y violenta el sentido democrático de la igualdad. Desde una perspectiva feminista, la lucha que dieron las mujeres (y los hombres) en el apogeo del momento socialdemócrata fue por la igualdad de condiciones socioeconómicas de todas y todos, es decir por un derecho universal. Asistir a las más pobres de las pobres, o sea hacer una distinción entre las que no son tan pobres y las que son extremadamente pobres, y hacerlo en nombre del "empoderamiento" de la mujer guiado por el discurso de equidad de género, en el contexto de un modelo de acumulación basado en la precarización laboral y social generalizada, no es para nada un logro feminista. Esta ha sido mi crítica fundamental a la institucionalización de discursos y prácticas feministas y de su mutación en prácticas de regulación en el ámbito del quehacer social del Estado. Desde un punto de vista de una política feminista, las políticas de focalización, apoyadas en un sistema contractual que invita a la supuesta participación de la sociedad civil a la implementación de programas -léase la involucración de una cantidad enorme de mujeres a nivel local, ya sea en sus barrios populares o en el municipio, como voluntarias, o en condiciones de precariedad laboral extremas, y en muchos casos invisibles - ha sido un modo muy efectivo de socavar la solidaridad social y, al mismo tiempo, de quebrar definitivamente la conflictiva y contradictoria solidaridad feminista que se urdió durante los ochenta. Mis trabajos durante los noventa y en el presente siglo detallan el proceso de transformación de estas relaciones entre mujeres y lo que he llamado la convergencia entre ciertos idearios y prácticas feministas y el proyecto de reestructuración neoliberal en curso ${ }^{18}$. Aquí querría sugerir también que poco a poco las investigaciones que he venido desarrollando en los últimos treinta años, y que consisten en interpretar estas transformaciones sociales y mutaciones del proyecto neoliberal, me han llevado últimamente a una

18 Véase Verónica Schild, “Market Citizenship and the ‘New Democracies': Ambiguous Legacies of Chilean Women's Movements"; "Die Freiheit der Frauen und gesellschaftlicher Fortschritt. Feministinen, der Staat und die Armen bei der Schaffung neoliberaler Gouvernementalitat", Peripherie: Zeitschrift fur Politik und Ökonomie in der Dritten Welt 93 (2003): 481-506; “NGOs, Feminist Politics and Neo-Liberal Latin American State Formations: Some Lessons from Chile", Canadian Journal of Development Studies 16, no. 4 (1995): 123-147. 
reflexión sobre la dimensión de género en lo que llamo la configuración de un Estado social neoliberalizado ${ }^{19}$.

L: Claro, eso podría ser como la pregunta inicial de los nudos neoliberales en términos del feminismo. Porque en el fondo es justamente esa idea de que las lógicas para tener un bien común, están en una lógica de competencia en relación a lo otro dentro de la misma comunidad, más que de una solidaridad para establecer un vínculo asociativo para el acceso o las demandas a ciertos derechos.

V: Precisamente. Aún más, ese vínculo asociativo es importante no sólo para acceder a ciertos derechos, sino para dar ese primer paso político que es poder articular demandas, formar alianzas, identificar prioridades, quiere decir, para hacer política feminista como práctica representativa e incluyente. A principios de los noventa, escuché frecuentemente a dirigentas feministas populares hablar de las "dueñas del género" para referirse a aquellas con las que urdieron complicidades durante los años de la dictadura, generalmente en el trabajo de las ONGs, y que ahora hablaban en nombre de todas, sin consultarles, y a partir de una agenda feminista institucional que según ellas las convertía en meras "clientas", y terminaba invisibilizandolas ${ }^{20}$.

L: Hay una crítica, pensado en la relación de las políticas de focalización en relación a las mujeres. El destinatario yo creo que - por lo menos en relación a la cuestión del cuidado- tiene que ver con las mujeres que trabajan remuneradamente. De esa manera, habría un reconocimiento en el sentido más capitalista clásico, de que el sujeto vale por lo que trabaja asalariadamente, no en relación a un reconocimiento de las mujeres a partir de su desarrollo personal, en relación a los tiempos de descanso, o de trabajo no remunerado etcétera. Entonces, lo pienso a través del empoderamiento como una retórica de mujeres que trabajan, que estudian, que se hacen cargo de la casa, y que aquello es valorado bajo una lógica que invisibiliza su explotación y precarización ¿Consideras que también esto ha jugado en contra del feminismo?

$\boldsymbol{V}$ : Claro que sí. Desde una perspectiva feminista crítica, las políticas públicas focalizadas no son realmente democráticas. A pesar de que el destinatario tiene que ver con las mujeres que trabajan, no todas las mujeres que trabajan tienen acceso a este "reconocimiento", sino que las de los quintiles más bajos. También es necesario reconocer que los contenidos y expectativas de ese empoderamiento, su dimensión cultural si tú quieres, son contradictorias. Por un lado, se proponen "empoderar" a las mujeres como entes autónomos y con proyectos de vida propios, algo que supuestamente se logra solamente en el mercado laboral, o desarrollando las habilidades para poder hacerlo (el así llamado capital social, que no hay que confundir con capacidades laborales mismas). Por el otro, vuelven a

19 Verónica Schild, "Geschlecht und Staat in Lateinamerika -die Zwei Gesichter Neoliberaler Regulation", en Staat in globaler Perspektive -neue und alte Entwicklungsstaaten, eds. HansJürgen Burchardt y Stefan Peters (Francfort/Nueva York: Campus-Verlag, 2015), 195-218.

20 Schild, "¿Nuevos Sujetos de Derechos?" 
reinscribirla como ente responsable por el cuidado de otros. Este es un tema que he analizado en mis trabajos y que resumo en publicaciones más recientes ${ }^{21}$. Entonces, el empoderamiento es tramposo, primero porque se limita a una visión mercantilizada y se vincula estrechamente a la inserción de la mujer al mercado: como emprendedora, como consumidora, y además como sujeto de endeudamiento responsable, que es el viraje actual en el contexto de la hiper-financialización de la economía. Además, las políticas interpelan a la mujer-madre, mujer-dueña de casa, hasta mujer responsable por la seguridad de su barrio, etcétera. Quiere decir, se responsabiliza a la mujer por el cuidado de los suyos y de su barrio, y esto cabe recordar es un tema clásico de las políticas asistencialistas.

Lo nuevo es que el Estado ya no juega el papel de proveedor responsable que apoya a la mujer dueña de casa y madre de sus hijos, un papel bastante mal cumplido en el caso de nuestra región, dadas las características particulares de nuestros capitalismos dependientes y de la enorme cantidad de mano de obra marginal. Ahora esa responsabilidad se le traspasa al mercado, y el Estado cumple un rol subsidiario, de apoyo, ofreciéndole empoderamiento a las mujeres para que salgan adelante por cuenta propia. Entonces, si eres realmente pobre, el Estado te ayuda a desplegar los talentos y atributos personales para insertarte en el mercado, y esta es una invitación como mujer autónoma, empoderada a hacerte responsable por ti misma y por el bienestar de la familia. La crítica que yo le hago a cierto análisis feministas que dice que lo que tenemos ahora es solamente una vuelta a reinscribir el tema mamá-mujer y el tema del cuidado, es que no dimensiona este cambio cultural. La figura clásica del Estado proveedor, tanto en su versión social demócrata como la del Estado benefactor en un país como Chile, era la de suplir el rol del marido irresponsable.

Como ya mencioné, el Estado alienta a las mujeres en nombre de una nueva femineidad, ahora como mujeres autónomas con "proyectos propios", pero lo que no se resuelve es el tema del cuidado. Esta sería, desde una perspectiva feminista, una doble inscripción si se quiere, que da por sentado que el tema del cuidado sigue siendo responsabilidad de las mujeres. Aquí hay que insertar eso si la dimensión de clase, entendida en su sentido relacional y racializado, ya que la cuestión del cuidado ha sido históricamente resuelta por muchas recurriendo al trabajo remunerado de otras. En el pasado, la inhabilidad del Estado benefactor para cubrir las necesidades de familias insertas en el sector informal de la economía forzó históricamente a muchas a salir a trabajar remuneradamente en aquellas actividades que seguían cumpliendo sin remuneración en su entorno familiar y su barrio. A pesar de que la participación de las mujeres en la fuerza de trabajo se ha incrementado en

21 Verónica Schild, "Feminismo y Neoliberalismo en América Latina", New Left Review 96 (2016): 63-79; "Rethinking Emancipation Beyond Neoliberal Regulation", Hypathia: A Journal of Feminist Philosophy 30, no. 3 (2015): 547-563. 
Chile y en toda la región, todos los indicadores muestran que el trabajo doméstico remunerado sigue siendo un componente importante de la ocupación de las mujeres y que, en muchos casos, no disminuye sino que va en aumento. Ahora bien, plantearse una alternativa a esta trampa que se le tiende a las mujeres en nombre de su empoderamiento personal, es hacer política feminista a partir de una respuesta más amplia al modelo de acumulación imperante y al neoliberalismo hegemónico. Esto significa necesariamente tejer alianzas con la multiplicidad de voces de mujeres y feministas que irrumpen con fuerza hoy día, y por necesidad, significa también hacer política anticapitalista desde una perspectiva del feminismo crítico.

L: Estimada Verónica, muchas gracias por compartir y dialogar sobre tus reflexiones y perspectivas. Sin duda nos interpelas desde el feminismo para seguir construyendo nuevas lecturas alternativas y divergentes en un contexto académico cada vez más neoliberalizado, e imbuido por una lectura y operación oficial del feminismo "académico".

\section{Referencias bibliográficas}

ANAMURI. "Anamuri: Celebrando 20 años de unidad y lucha de las mujeres del campo". Medio a Medio, 13 de junio de 2018. Consultado en agosto de 2018, disponible en http://www.agenciadenoticias.org/anamuri-celebrando-20anos-de-unidad-y-lucha-de-las-mujeres-del-campo/.

Barrig, Maruja . "Los malestares del feminismo latinoamericano: una nueva lectura", ponencia presentada en la conferencia LASA, 1998. Manuscrito.

Brown, Wendy. Undoing the Demos. Neoliberal's Stealth Revolution. Nueva York: Zone Books, 2015.

Clarke, John. Changing Welfare, Changing States. Londres: Sage Publications, 2004.

Follegati, Luna. "El feminismo se ha vuelto una necesidad: movimiento estudiantil y organización feminista (2000-2016)". En Juventud y espacio en las Américas. I Taller Casa Tomada, editado por Ana Niria Albo y Camila Valdés León La Habana: Editorial Casa de las Américas, 2016.

Painemal, Millaray e Isabel Cañet. “¿Es que acaso debemos ser todas feministas? Reflexiones de mujeres mapuche para un debate". El Desconcierto, 04 de marzo de 2018. Consultado el 13 de agosto de 2018, disponible en http://www. eldesconcierto.cl/2018/03/04/es-que-acaso-debemos-ser-todas-feministasreflexiones-de-mujeres-mapuche-para-un-debate/.

Pozo, Hernán. "Mujeres Latinoamericanas en Cifras, avances de investigación”. VI. Situación Jurídica de la Mujer. Documento de Trabajo. Serie: Estudios Sociales (16). Santiago de Chile: FLACSO, 1991. 
Rojas, Claudia. "Poder, mujeres y cambio en chile (1964-1973): un capítulo de nuestra historia". Tesis para optar al grado de magíster en Historia, Universidad Autónoma Metropolitana, 1994.

Schild, Verónica. "Feminismo y neoliberalismo en América Latina", New Left Review 96 (2016): 63-79.

"Rethinking Emancipation Beyond Neoliberal Regulation". Hypathia: A Journal of Feminist Philosophy 30, no. 3 (2015): 547-563.

"Geschlecht und Staat in Lateinamerika - die Zwei Gesichter Neoliberaler Regulation", en Staat in globaler Perspektive - neue und alte Entwicklungsstaaten, editado por Hans-Jürgen Burchardt y Stefan Peters (Francfort/Nueva York: Campus-Verlag, 2015), 195-218.

"Neo-Liberalism's New Market Citizens: The Civilizing Dimension of Social Programs in Chile". Citizenship Studies 4 (2000): 275-305.

"Die Freiheit der Frauen und gesellschaftlicher Fortschritt. Feministinen, der Staat und die Armen bei der Schaffung neoliberaler Gouvernementalitat". Peripherie: Zeitschrift fur Politik und Ökonomie in der Dritten Welt 93 (2003): 481-506.

"Market Citizenship and the 'New Democracies': The Ambiguous Legacies of Contemporary Chilean Women's Movements". Social Politics: International Studies in Gender, State \& Society 5, no. 2 (1998): 232-249.

"NGOs, Feminist Politics and Neo-Liberal Latin American State Formations: Some Lessons from Chile". Canadian Fournal of Development Studies 16, no. 4 (1995):123-147.

“¿Nuevos Sujetos de Derechos? Movimientos de mujeres y la construcción de ciudadanía en las "nuevas democracias". En Política cultural \& cultura política: Una nueva mirada sobre los movimientos sociales latinoamericanos, editado por Arturo Escobar, Sonia Álvarez y Evelina Dagnino, 119-146. Bogotá: Taurus-ICANH, 2001.

"Recasting 'Popular' Movements: Gender and Political Learning in Neighbourhood Organizations in Chile". Latin American Perspectives 21 (1994): 59-80.

"Care and Punishment in Latin America: The Gendered Neoliberalization of the Chilean State." En Neoliberalism Interrupted: Social Change and Contested Governance in Contemporary Latin America, editado por Mark Goodale y Nancy Postero. Stanford: Stanford University Press, 2013.

- "Die Freiheit der Frauen und gesellschaftlicher Fortschritt. Feministinen, der Staat und die Armen bei der Schaffung neoliberaler Gouvernementalitat." Peripherie: Zeitschrift fur Politik und Ökonomie in der Dritten Welt 93 (2003): 481506. 
. "Empowering Consumer Citizens or Governing Poor Female Subjects. The Institutionalization of 'Self-Development' in the Chilean Social Policy Field." Fournal of Consumer Culture 7, no. 2 (2007): 179-203.

"Feminismo y Neoliberalismo en América Latina." New Left Review 96 (2016): 63-79.

- "Geschlecht und Staat in Lateinamerika - die Zwei Gesichter Neoliberaler Regulation". En Staat in globaler Perspektive - neue und alte Entwicklungsstaaten, editado por Hans-Jürgen Burchardt y Stefan Peters. Frankfurt/New York: Campus-Verlag, 2015.

- "Los feminismos y la construcción del estado social neoliberal en América Latina". En Desigualdades en un Mundo Globalizado, editado por Narda Henriquez, Gerardo Damonte, Marianne Braig y Barbara Göbel. Lima: CISEPA Pontificia Universidad Católica del Perú, 2015.

- "Rethinking Emancipation Beyond Neoliberal Regulation." Hypathia Fournal of Feminist Philosophy 30, no. 3 (2015): 547-563.

Svampa, Maristella. La sociedad excluyente: La Argentina bajo el signo del neoliberalismo. Buenos Aires, Editorial Taurus: 2005.

Verónica Schild. Profesora emérita de la Universidad de Western (Ontario, Canadá). Nacida en Chile, sus estudios universitarios de pre y postgrado los realizó en Estados Unidos y Canadá. Es Ph.D. en Ciencia Política por la Universidad de Toronto (1991), M.A. en teoría de la educación por la Universidad de Toronto (1982) y B.A. por la Universidad George Mason (1978). Cuenta con una destacada trayectoria en el campo de la investigación comparativa sobre política latinoamericana, con énfasis en temas feministas, gubernamentalidad neoliberal y ciudadanía. Ha investigado por más de treinta años la realidad latinoamericana desde la perspectiva feminista del conocimiento situado, analizando con detención el caso de Chile en el contexto y auge del neoliberalismo. Fue Directora del Centre for the Study of Theory and Criticism de la Universidad de Western, Ontario, y ha sido profesora visitante e investigadora en destacadas universidades europeas y latinoamericanas. Sus publicaciones son múltiples, realizando un aporte sustantivo a la comprensión actual del feminismo y su cruce con las políticas públicas bajo un contexto democrático y neoliberal. Correo electrónico: vschild@uwo.ca.

Luna Follegati Montenegro. Candidata a doctora en Filosofía Política por la Universidad de Chile (Santiago, Chile). Magíster en Comunicación Política y Licenciada en Historia por la Universidad de Chile. Sus investigaciones se vinculan a la teoría e historia de las transiciones a la democracia, teorías de género y movimiento feminista. Ha trabajado en el ámbito de la docencia en diversas 
instituciones académicas (Universidad de Chile, Universidad de Valparaíso, USACH, UMCE). Publicó junto a Rodrigo Karmy Estudios En Gubernamentalidad. Ensayos sobre poder, vida y neoliberalismo, de la editorial Communes (2018). Sus artículos más recientes son "El feminismo se ha vuelto una necesidad: movimiento estudiantil y organización feminista en Chile (2000-2017)", Revista Anales de la Universidad de Chile 14/7 (2018) y de "El constante aparecer del movimiento feminista. Reflexiones desde la contingencia", en Mayo Feminista. La Rebelión contra el patriarcado, editado por Faride Zerán (Santiago: Lom, 2018). Correo electrónico: Ifollegati@gmail.com. 